\title{
A Rare Case of Testicular Torsion in a 75 Year Old.
}

\author{
Dr. Narang $\mathrm{Naku}^{1}$, Dr. Balasubrahmanya K. S ${ }^{2}$, Dr. Manjunath R.D ${ }^{3}$, \\ Dr. Anil Raj.D ${ }^{4}$ \\ ${ }^{I}$ Resident,Department of General Surgery, Mysore Medical College \& Research Institute, Mysore. \\ ${ }^{2}$ Associate professor,Department of General Surgery, Mysore Medical College \& Research Institute,Mysore. \\ ${ }^{3}$ Assistant professor,Department of General Surgery, Mysore Medical College \& Research Institute, Mysore. \\ ${ }^{4}$ Resident,Department of General Surgery,Mysore Medical College \& Research Institute, Mysore.
}

\begin{abstract}
:
Background: Testicular torsion occurs when a_testicle torts on the spermatic cord resulting in the cutting off of blood supply. The most common symptom is acute testicular pain and the most common underlying cause, a bell-clapper deformity. Testicular torsion implies obstruction of first venous, and later, arterial flow. The extent of testicular ischemia will depend on the degree of twisting $\left(180^{\circ}-720^{\circ}\right)$ and the duration of the torsion. Testicular salvage is more likely in patients treated within 4-6 hours after the onset of torsion.

Case Presentation: We came across an unusual case of testicular torsion in a 75 years old man, who presented to our emergency department with a 7 days history of right-sided testicular pain with no pyrexia,urinary symptoms and negative urine routine, initially treated with antibiotics elsewhere as torsion amongst the elderly population is rarely described. This case presents the oldest surgically confirmed case of testicular torsion, in a 75-year-old male, documented in a journal so far . There was no pyrexia or urinary symptoms and negative urine routine.

In adults above the age of 40, likely diagnoses include epididymo-orchitis, epididymitis, neoplasm or hydrocele. Clinical differentiation with epididymo-orchitis can be difficult in any age range. Clinical signs such as fever, elevated C-reactive protein and positive urine test are suggestive of epididymo-orchitis/orchitis.

Conclusion: This case study demonstrates that testicular torsion can occur at any age, and clinical suspicion should always be high in patients presenting with testicular pain and a negative urine tests, regardless of age. Although risk in this subgroup is low, the identification of a potentially reversible testicular abnormality should be of high priority.
\end{abstract}

Key words: Testicular torsion, bell-clapper deformity,testicular ischemia, twisting degree (180 - 720 ), testicular salvage, old age (75 yrs),epididymo-orchitis,epididymitis, neoplasm, hydrocele.

\section{Introduction}

Torsion amongst the elderly population is rarely described.This case presents the oldest surgically confirmed case of testicular torsion, in a 75-year-old male,documented in a journal so, far .

\section{Case Report}

A 75-year-old male presented to the emergency department with a 7-days history of right-sided testicular pain. Symptoms were of gradual onset, and were initially treated with antibiotics from his general practitioner. There was no history of trauma, injury, pyrexia or voiding symptoms. Urine dipstick test was negative. Examination revealed an acutely tender right groin and right testicle with a markedly enlarged, erythematous right hemiscrotum. Antibiotics were changed to doxycycline from ciprofloxacin,and the patient was referred for an urgent ultrasound which was performed the same day. Full blood count revealed white cell count of 9.3_109/L with a C-reactive protein (CRP) of $54 \mathrm{mg} / \mathrm{L}$. All other blood tests were normal. An ultrasound scan revealed no vascularity within the right testicle. The patient was taken for scrotal exploration under general anaesthetic. This revealed a torted right testicle with pus present. A right orchidectomy was performed along with left-sided testicular fixation. The patient had an uneventful post-operative course, and was discharged home the following day.

\section{Discussion}

Torsion as a cause for testicular pain in elderly men is rare.It is believed to be due to an anatomical bell-clapper deformity of the testicle [1] and with this genetic disposition, $65 \%$, of cases usually present at 18 years of age [2]. However, other possible causes of torsion have been recognised such as trauma and increasedtesticular volume [3]. In adults above the age of 40, likely diagnoses include epididymo-orchitis, epididymitis, neoplasm or hydrocele. Many of the patients in this age range do not require imaging, and are sent home on antibiotics. Clinical differentiation with epididymo-orchitis can be difficult in any age range. Clinical signs such 
as fever, elevated CRP and positive urine dipstick test for leukocytes and nitrites [4] are suggestive of epididymo-orchitis/orchitis. Therefore in the absence of this, alternative diagnosis should be explored more thoroughly at an early opportunity. As studies suggest that testicular viability in a true torsion is reliant on time to presentation and subsequent definitive surgery, early diagnosis is paramount in order to reduce consequential loss of organ, cosmetic effects and minimize the possibility of reduced fertility [5]. Testicular infarction is as high as $80 \% 10 \mathrm{~h}$ after the onset of pain [6], and therefore ultrasound with Doppler should not be used in the likelihood of torsion if this delays surgical intervention. Despite this, as it has been shown to offer an overall accuracy of 97\% [7], it is becoming increasingly used in acute presentations of acute scrotal pain where clinical suspicion is low.

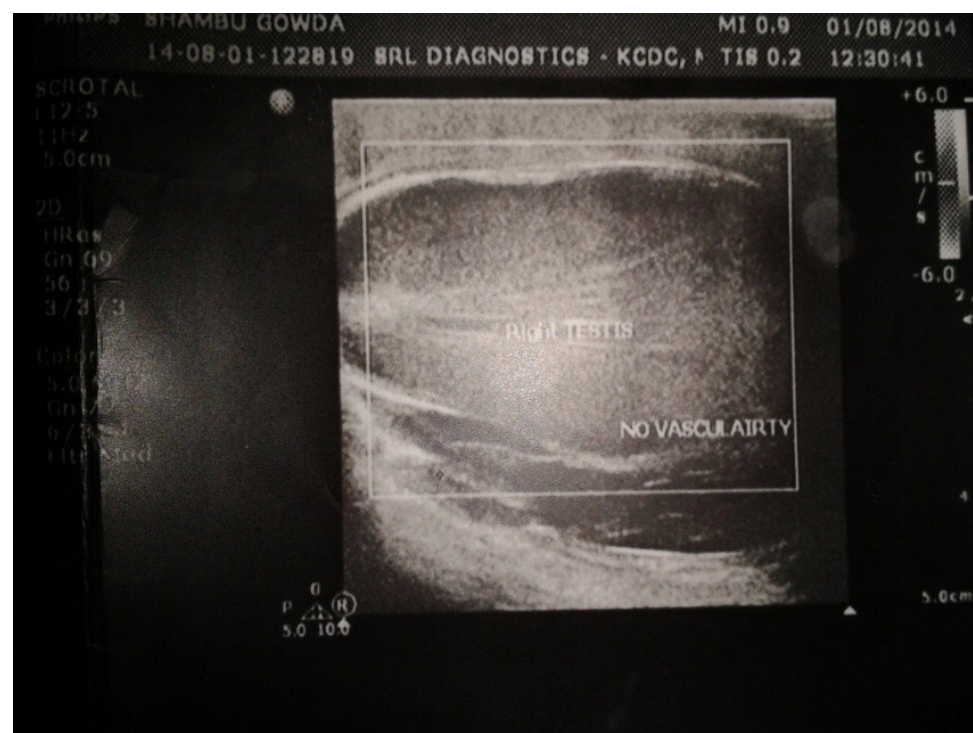

Ultra sound report showing avascularity of right testis, epididymal body and tail -ISCHEMIA.

\section{Per-Operative Findings - Videos And Images ;}

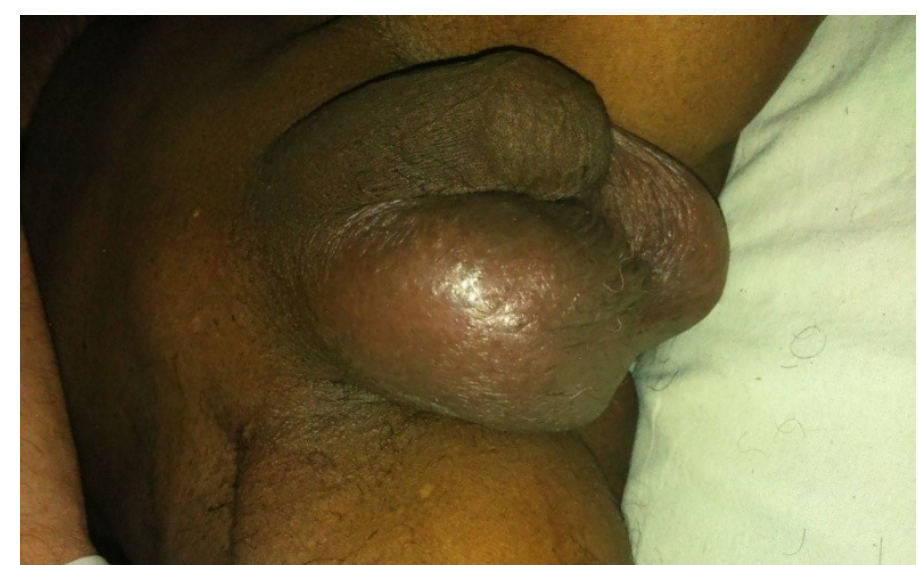

ON table pre-operative appearance of torted testis ; tensed, stretched skin with abnormally placed torted testis . 

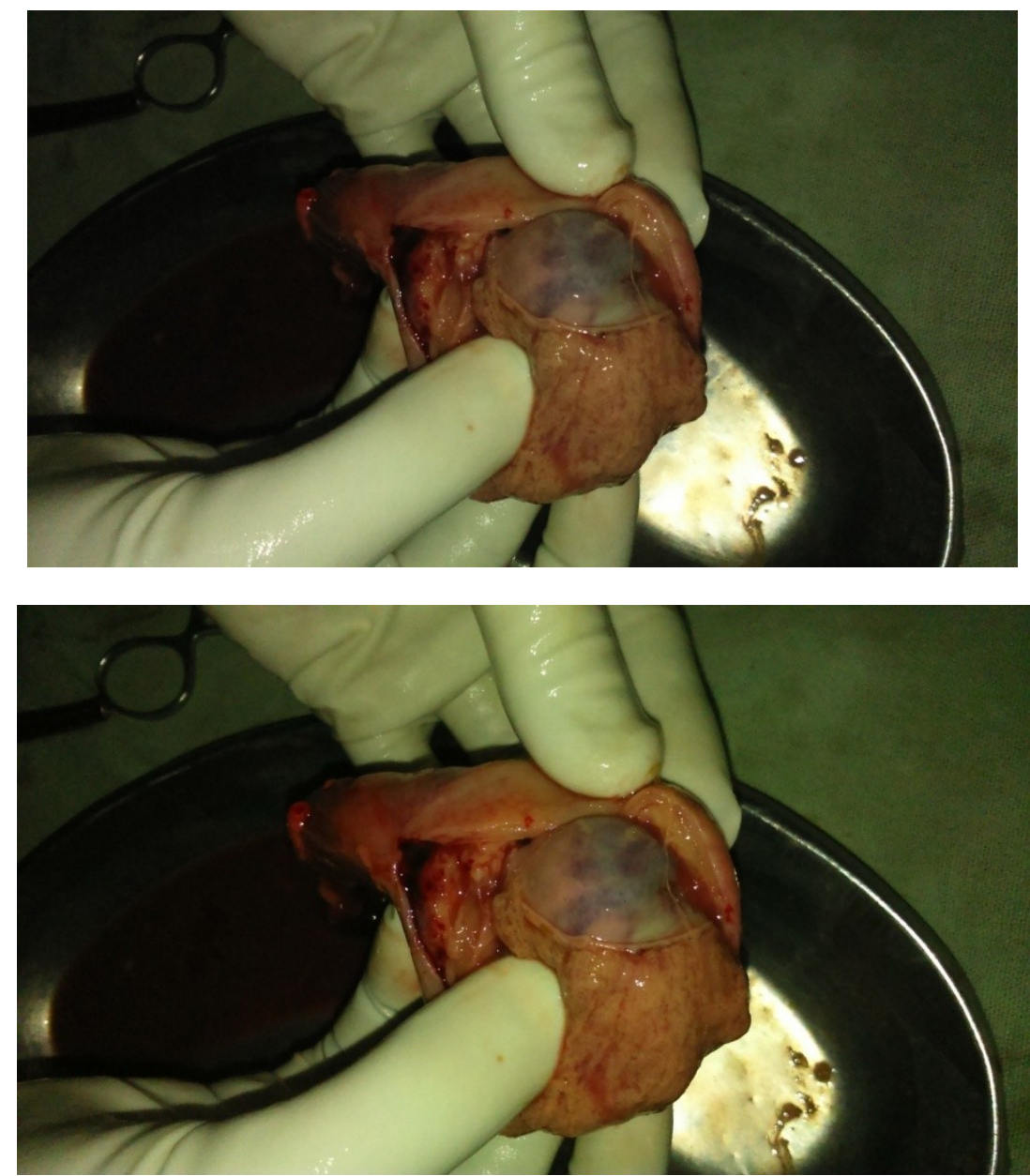

TORTED TESTIS per operative visble ischaemic changes.

\section{Conclusion}

This case study demonstrates that testicular torsion can occur at any age, and clinical suspicion should always be high in patients presenting with testicular pain and negative urine dipstick regardless of age. Although risk in this subgroup is low, the identification of a potentially reversible testicular abnormality should be of high priority.

\section{References}

[1]. Alfert HJ, Canning DA. Testicular torsion in a 62 year old man. J Urol 1987;138:149-50

[2]. Edelsberg JS, Surh YS. The acute scrotum. Emerg Med Clin North Am 1988;6:521-46.

[3]. Arce JD, Cortes M, Vargas JC. Sonographic diagnosis of acute spermatic cord torsion. Rotation of the cord: a key to the diagnosis. Pediatr Radiol 2002;32:485-91.

[4]. Chiang MC, Chen HW, Fu RH, Lien R, Wang TM, Hsu JF. Clinical features of testicular torsion and epididymo-orchitis in infants younger than 3 months. J Pediatr Surg 2007;42:1574-7.

[5]. Dunne P, O'Loughlin B. Testicular torsion: time is the enemy. ANZ J Surg 2000;70:441-2.

[6]. Jaison A, Mitra B, Cameron P, Sengupta S. Use of ultrasound and surgery in adults with acute scrotal pain. ANZ J Surg 2011;81:366-70

[7]. Burks D, Markey B, Burkhard T, et al. Suspected testicular torsion and ischemia: evaluation with color Doppler sonography. Radiology 1990;175:815-21. 